\title{
Palladium-catalysed ortho arylation of acetanilides
}

\author{
GUO-ZHEN ZHANG, CHENG-QUN CHEN, XIN-HUA FENG and GUO-SHENG HUANG* \\ State Key Laboratory of Applied Organic Chemistry, Lanzhou 73000, P.R. China \\ e-mail: hgs2368@163.com
}

MS received 12 January 2009; revised 20 July 2009; accepted 14 September 2009

\begin{abstract}
The palladium-catalysed direct arylation of acetanilides by using $\mathrm{C}-\mathrm{H}$ activation methodology has been demonstrated. Several acetanilides were coupled with aryl iodides in the presence of $10 \mathrm{~mol} \%$ of $\mathrm{Pd}(\mathrm{OAc})_{2}, 1.0$ equiv of $\mathrm{Cu}(\mathrm{OTf})_{2}$, and 0.6 equiv of $\mathrm{Ag}_{2} \mathrm{O}$ to afford the corresponding products in moderate to excellent yields. The results showed that the amount of $\mathrm{Ag}_{2} \mathrm{O}$ was important for this protocol.
\end{abstract}

Keywords. Palladium; acetanilides; $\mathrm{C}-\mathrm{H}$ activation; aryl iodides; 2-arylacetanilides; 2,6-diarylacetanilides.

\section{Introduction}

Aryl-aryl bond formation plays an important role in the area of modern organic synthesis. These bonds are common in natural products, pharmaceuticals, catalyst ligands, and materials. ${ }^{1}$ Traditionally, transition metalcatalysed cross-coupling reactions of organometallic reagents and aryl halides or pseudohalides constitute one of the most useful methods for the synthesis of biaryl molecules. However, these strategies, requiring installation of functionality on both coupling components, are neither atom economic nor green. ${ }^{2} \mathrm{C}-\mathrm{H}$ functionalization is the most sustainable and straight-forward method to construct complicated structures and has received significant attention in the past several decades. ${ }^{3}$ Various functional groups containing heteroatoms such as acetoamino, ${ }^{4}$ oxazolyl, ${ }^{5}$ pyridyl, ${ }^{6}$ or imino ${ }^{7}$ groups act as directing groups, and influence the regioselectivity of the reaction. With the help of acetamido group, ortho $\mathrm{C}-\mathrm{H}$ of acetanilide could be functionlized ${ }^{8}$ regioselectively. Shi and coworkers have developed palladium-catalysed ortho-arylation of amides with aryl boronic acids ${ }^{8 f}$ and trialkoxysilanes, ${ }^{8 g}$ using $\mathrm{Cu}(\mathrm{OTf})_{2}$ and $\operatorname{Ag}(\mathrm{I})$ as stoichiometric oxidants. Daugulis has reported coupling reactions of pivalanilides with aryl iodides using a catalytic amount of $\mathrm{Pd}(\mathrm{OAc})_{2}$ and 1 equivalent of $\mathrm{Ag}(\mathrm{OAc})_{2}$ as an additive ${ }^{8 \mathrm{i}}$ In this method, highly acidic $\mathrm{CF}_{3} \mathrm{COOH}$ must be used as solvent, and only 2,6-diarylpivalanilides

*For correspondence were obtained when pivalanilide and 4-substituted pivalanilides were used as substrates. Inspired by these results, we report here ortho arylation of acetanilide with aryl iodides in the presence of $\mathrm{Pd}(\mathrm{OAc})_{2}$ $(10 \mathrm{~mol} \%), \quad \mathrm{Cu}(\mathrm{OTf})_{2} \quad$ ( 1.0 equiv), and $\mathrm{Ag}_{2} \mathrm{O}$ ( $0 \cdot 6$ equiv).

\section{Experimental}

NMR spectra were recorded on a Mercury 4N-PEG$300\left({ }^{1} \mathrm{H}: 300 \mathrm{MHz} ;{ }^{13} \mathrm{C}: 75 \mathrm{MHz}\right.$ ) spectrometer, using $\mathrm{CDCl}_{3}$ as a solvent and TMS as the internal standard. IR spectra were recorded on Nicolet Nexus 670 FT$\mathrm{TR}$ spectrophotometer in $\mathrm{KBr}$ pellets or $\mathrm{KBr}$ film. Mass spectra were recorded by the EI method on a HP 5998 mass spectrometer.

\subsection{General experimental procedure}

A mixture of acetanilide $\mathbf{1}(0.2 \mathrm{mmol})$, aryl iodide $\mathbf{2}$ (1 mmol 5.0 equiv), $\mathrm{Pd}(\mathrm{OAc})_{2} \quad(0.02 \mathrm{mmol}, \quad 0.10$ equiv), anhydrous $\mathrm{Cu}(\mathrm{OTf})_{2}(0.2 \mathrm{mmol}, 1.0$ equiv), $\mathrm{Ag}_{2} \mathrm{O} \quad(0.12 \mathrm{mmol}, 0.6$ equiv. $)$ and dried 1,2dichloroethane $(2 \mathrm{ml})$ was added to a $14 \times 90 \mathrm{~mm}$ glass test tube. The tube was sealed with a rubber plug, and the reaction mixture was stirred and heated at $90^{\circ} \mathrm{C}$ in an oil bath. The progress of the reaction was monitored by thin-layer chromatography. After completion, the reaction mixture was poured into an excess of water and extracted with $\mathrm{CH}_{2} \mathrm{Cl}_{2}$ $(3 \times 1 \mathrm{ml})$. The organic phases were dried and the 
solvent was evaporated. The residue was purified by preparative TLC using petroleum ether-EtOAc $(8: 1)$ as the eluent to afford the desired coupled products.

\section{1a N-(4,5-Dimethyl-biphenyl-2-yl)-acetamide}

(3aa): White solid; m.p.: $120-121{ }^{\circ} \mathrm{C} ;{ }^{1} \mathrm{H}$ NMR $\left(300 \mathrm{MHz}, \mathrm{CDCl}_{3}\right): \delta=7.95(s, 1 \mathrm{H}, \mathrm{NH}), 7 \cdot 45-7.34$ $(m, 5 \mathrm{H}), 7.05(d, J=12.30 \mathrm{~Hz}, 2 \mathrm{H}), 2.30(s, 3 \mathrm{H})$, $2.26(s, 3 \mathrm{H}), 2.00(s, 3 \mathrm{H}) ;{ }^{13} \mathrm{C}$ NMR $(75 \mathrm{MHz}$, $\left.\mathrm{CDCl}_{3}\right): \delta=168 \cdot 26,138 \cdot 31,136 \cdot 71,132.93,132 \cdot 05$, $131.01, \quad 130.34, \quad 129 \cdot 17, \quad 128.83, \quad 127.53,123 \cdot 47$, 24.33, 19.69, 19.15; IR ( $\mathrm{KBr}$ plate, $\mathrm{CDCl}_{3}, \mathrm{~cm}^{-1}$ ) $v=3276,3025,1667,1521$; MS (EI): $m / z=239$, 197. Anal. Calcd. for $\mathrm{C}_{16} \mathrm{H}_{17} \mathrm{NO}: \mathrm{C}, 80 \cdot 30 ; \mathrm{H}, 7 \cdot 16$; N, 5.85. Found: C, 80.48; H, 7.30; N, 5.92.

$2.1 \mathrm{~b} N$-(5-Chloro-biphenyl-2-yl)-acetamide (3ba): White solid; m.p.: $115-116^{\circ} \mathrm{C} ;{ }^{1} \mathrm{H}$ NMR $(300 \mathrm{MHz}$, $\left.\mathrm{CDCl}_{3}\right): \delta=8.19(d, J=8.70 \mathrm{~Hz}, 1 \mathrm{H}, \mathrm{NH}), 7.50$ $7 \cdot 39(m, 3 \mathrm{H}), 7 \cdot 33-7 \cdot 27(m, 3 \mathrm{H}), 7 \cdot 22-7 \cdot 16(m, 2 \mathrm{H})$, $1.94(s, 3 \mathrm{H}) ;{ }^{13} \mathrm{C}$ NMR $\left(75 \mathrm{MHz}, \mathrm{CDCl}_{3}\right)$ : $\delta=168 \cdot 17,136 \cdot 77,133 \cdot 72,133 \cdot 27,129 \cdot 71,129 \cdot 16$, $128.93,128.40,128.13,122.92,24.41$; IR $(\mathrm{KBr}$ plate, $\left.\mathrm{CDCl}_{3}, \mathrm{~cm}^{-1}\right) v=3267,2920,1666,1517$; MS (EI): $m / z=247,245,203$. Anal. Calcd. for $\mathrm{C}_{14} \mathrm{H}_{12}$ CINO: C, 68.44; H, 4.92; N, 5.70. Found: C, 68.61; $\mathrm{H}, 4 \cdot 79 ; \mathrm{N}, 5 \cdot 54$.

2.1c N-Acetyl-4-chloro-2,6-diphenyl-aniline (4ba): White solid; m.p.: $176-177^{\circ} \mathrm{C}{ }^{1} \mathrm{H}$ NMR $(300 \mathrm{MHz}$, $\left.\mathrm{CDCl}_{3}\right): \delta=7.43-7.35(m, 12 \mathrm{H}), 6.51(s, 1 \mathrm{H}, \mathrm{NH})$, $1.68(s, 3 \mathrm{H}) ;{ }^{13} \mathrm{C}$ NMR $\left(75 \mathrm{MHz}, \mathrm{CDCl}_{3}\right): \delta=68.39$, $142 \cdot 50,138.55,133 \cdot 16,130 \cdot 00,129 \cdot 65,128.56$, $128.33,127.79,22.69$; IR ( $\mathrm{KBr}$ plate, $\mathrm{CDCl}_{3}$, $\left.\mathrm{cm}^{-1}\right) \quad v=3228, \quad 2924, \quad 1655, \quad 1515 ; \quad \mathrm{MS}$ (EI): $m / z=323,321,279$. Anal. Calcd. for $\mathrm{C}_{20} \mathrm{H}_{16} \mathrm{ClNO}$ : C, 74.65; H, 5.01; N, 4.35. Found: C, 74.71; H, $5 \cdot 11 ; \mathrm{N}, 4 \cdot 28$.

2.1d N-(3-Methyl-biphenyl-2-yl)-acetamide (3ca): White solid; m.p.: $106-108^{\circ} \mathrm{C} ;{ }^{1} \mathrm{H}$ NMR $(300 \mathrm{MHz}$, $\left.\mathrm{CDCl}_{3}\right): \delta=7 \cdot 41-7 \cdot 22(m, 7 \mathrm{H}), 7 \cdot 17-7 \cdot 14(m, 1 \mathrm{H})$, $6.98(s, 1 \mathrm{H}, \mathrm{NH}), 2.27(s, 3 \mathrm{H}), 1.93(s, 3 \mathrm{H}) \cdot{ }^{13} \mathrm{C}$ NMR (75 MHz, $\left.\mathrm{CDCl}_{3}\right): \delta=169.43,139.57,139.52$, $136 \cdot 70,132.57,129.95,128.72,128 \cdot 15,127 \cdot 76$, 127.27, 127.18, 22.77, 18.47; IR ( $\mathrm{KBr}$ plate, $\mathrm{CDCl}_{3}$, $\left.\mathrm{cm}^{-1}\right) \quad v=3257, \quad 3027, \quad 1660, \quad 1524 ; \quad \mathrm{MS}$ (EI): $m / z=225$, 183. Anal. Calcd. for $\mathrm{C}_{15} \mathrm{H}_{15} \mathrm{NO}$ : C, 79.97; H, 6.71; N, 6.22. Found: C, 80.15; H, 6.54; $\mathrm{N}, 6 \cdot 39$. 2.1e N-(4-Chloro-biphenyl-2-yl)-acetamide (3ea): White solid; m.p.: $108-109^{\circ} \mathrm{C} ;{ }^{1} \mathrm{H}$ NMR $(300 \mathrm{MHz}$, $\left.\mathrm{CDCl}_{3}\right): \delta=8.37(s, 1 \mathrm{H}, \mathrm{NH}), 7.51-7.42(m, 3 \mathrm{H})$, 7.34-7.30 (m, 2H), 7.24-7.14 (m, 1H), 7.11 $(s, 2 \mathrm{H})$, $2 \cdot 00(s, 3 \mathrm{H}) ;{ }^{13} \mathrm{C}$ NMR $\left(75 \mathrm{MHz}, \mathrm{CDCl}_{3}\right): \delta=168 \cdot 16$, $137.00,135.63,133.94,130.84,130.16,129.22$, 129.06, 128.27, 124.20, 121.21 24.55. IR (KBr plate, $\left.\mathrm{CDCl}_{3}, \mathrm{~cm}^{-1}\right) v=3278,2919,1673,1516$; MS (EI): $m / z=247,245,203$. Anal. Calcd. for $\mathrm{C}_{14} \mathrm{H}_{12} \mathrm{ClNO}$ : C, 68.44; H, 4.92; N, 5.70. Found: C, 68.29; H, $4 \cdot 99 ; \mathrm{N}, 5 \cdot 85$.

2.1f N-Biphenyl-2-yl-acetamide (3fa): White solid; m.p.: $104-105^{\circ} \mathrm{C} ;{ }^{1} \mathrm{H}$ NMR (300 $\mathrm{MHz}, \mathrm{CDCl}_{3}$ ): $\delta=8.26(d, \mathbf{J}=8.10 \mathrm{~Hz}, 1 \mathrm{H}, \mathrm{NH}), 7.51-7.34(m$, $6 \mathrm{H}), 7.25-7.13(\mathrm{~m}, 3 \mathrm{H}), 2.02(\mathrm{~s}, 3 \mathrm{H}) ;{ }^{13} \mathrm{C} \mathrm{NMR}$ $\left(75 \mathrm{MHz}, \mathrm{CDCl}_{3}\right): \quad \delta=168 \cdot 19, \quad 138.07, \quad 134.55$, $132.34,129.94,129.06,128.91,128.22,127.79$, $124 \cdot 32,121 \cdot 84,24 \cdot 33$; IR ( $\mathrm{KBr}$ plate, $\mathrm{CDCl}_{3}, \mathrm{~cm}^{-1}$ ) $v=3288,3028,1661,1532$; MS (EI): $m / z=211$, 169. Anal. Calcd. for $\mathrm{C}_{14} \mathrm{H}_{13} \mathrm{NO}$ : $\mathrm{C}, 79.59 ; \mathrm{H}, 6.20$; N, 6.63. Found: C, 79.41; H, 6.38; N, 6.47.

2.1g N-Acetyl-2,6-diphenyl-aniline (4fa): White solid; m.p.: $228-230^{\circ} \mathrm{C} ;{ }^{1} \mathrm{H}$ NMR $\left(300 \mathrm{MHz}, \mathrm{CDCl}_{3}\right)$ : $\delta=7.45-7.33(m, 13 \mathrm{H}), 6.73(s, 1 \mathrm{H}, \mathrm{NH}), 1.65(s$, $3 \mathrm{H}) ;{ }^{13} \mathrm{C}$ NMR $\left(75 \mathrm{MHz}, \mathrm{CDCl}_{3}\right): \delta=169 \cdot 36,140 \cdot 89$, $139.76,129.85,129 \cdot 11,128 \cdot 70,128 \cdot 10,127 \cdot 65$, 127.21, 22.70; IR ( $\mathrm{KBr}$ plate, $\left.\mathrm{CDCl}_{3}, \mathrm{~cm}^{-1}\right) v=3232$, $3025,1653,1522$; MS (EI): $m / z=287,245$. Anal. Calcd. for $\mathrm{C}_{20} \mathrm{H}_{17} \mathrm{NO}$ : C, 83.59; H, 5.96; N, 4.87. Found: C, 83.44; H, 6.14; N, 5.01.

2.1h N-(5-Methyl-biphenyl-2-yl)-acetamide (3ga): White solid; m.p.: $104-106^{\circ} \mathrm{C} ;{ }^{1} \mathrm{H}$ NMR $(300 \mathrm{MHz}$, $\left.\mathrm{CDCl}_{3}\right): \delta=8.06(d, J=8.40 \mathrm{~Hz}, 1 \mathrm{H}, \mathrm{NH}), 7.48-$ $7.33(m, 5 \mathrm{H}), 7.16(d, J=8.40 \mathrm{~Hz}, 1 \mathrm{H}), 7.05(s, 2 \mathrm{H})$, $2.27(s, 3 \mathrm{H}), 1.99(s, 3 \mathrm{H}) ;{ }^{13} \mathrm{C}$ NMR $(75 \mathrm{MHz}$, $\left.\mathrm{CDCl}_{3}\right): \delta=168 \cdot 19,138 \cdot 34,134 \cdot 06,132 \cdot 50,132 \cdot 05$, $130.58,129.13,128.91,128 \cdot 87,127 \cdot 76,122 \cdot 06$, 24.38, 20.80; IR ( $\mathrm{KBr}$ plate, $\left.\mathrm{CDCl}_{3}, \mathrm{~cm}^{-1}\right) \quad v=3271$, 3028, 1666, 1518; MS (EI): $m / z=225,183$. Anal. Calcd. for $\mathrm{C}_{15} \mathrm{H}_{15} \mathrm{NO}$ : C, 79.97; H, 6.71; N, 6.22. Found: C, 79.79; H, 6.89; N, 6.12.

2.1i N-Acetyl-4-methyl-2,6-diphenyl-aniline (4ga): White solid; mp: $206-208^{\circ} \mathrm{C} ;{ }^{1} \mathrm{H}$ NMR $(300 \mathrm{MHz}$, $\left.\mathrm{CDCl}_{3}\right): \delta=7 \cdot 40-7.32(m, 10 \mathrm{H}), 7 \cdot 19(s, 2 \mathrm{H}), 6.53$ $(s, 1 \mathrm{H}, \mathrm{NH}), 2.42(s, 3 \mathrm{H}), 1.63(s, 3 \mathrm{H}) ;{ }^{13} \mathrm{C} \mathrm{NMR}$ $\left(75 \mathrm{MHz}, \quad \mathrm{CDCl}_{3}\right): \quad \delta=169.61, \quad 140.81, \quad 139.90$, $137.52,130.61,129 \cdot 11,128.73,128.60,127 \cdot 19$, 
22.79, 21.05; IR ( $\mathrm{KBr}$ plate, $\left.\mathrm{CDCl}_{3}, \mathrm{~cm}^{-1}\right) v=3275$, 3023, 1658, 1523; MS (EI): $m / z=301,259$. Anal Calcd. for $\mathrm{C}_{21} \mathrm{H}_{19} \mathrm{NO}$ : C, 83.69; H, 6.35; N, 4.65. Found: C, 83.85 H, 6.19; N, 4.74.

2.1j N-(3-Methoxy-4'-methyl-biphenyl-2-yl)-acetamide (3hb): White solid; m.p.: ${ }^{124}-125^{\circ} \mathrm{C} ;{ }^{1} \mathrm{H}$ NMR $\left(300 \mathrm{MHz}, \mathrm{CDCl}_{3}\right): \delta=7 \cdot 26-7 \cdot 18(m, 5 \mathrm{H})$, $6.93(d, J=8.40 \mathrm{~Hz}, 2 \mathrm{H}), 6.66(s, 1 \mathrm{H}, \mathrm{NH}), 3.87(s$, $3 \mathrm{H}), 2.40(s, 3 \mathrm{H}), 2.00(s, 2 \mathrm{H}), 1.68(s, 1 \mathrm{H}) ;{ }^{13} \mathrm{C}$ NMR (75 MHz, $\left.\mathrm{CDCl}_{3}\right): \delta=169 \cdot 50,155.09,140 \cdot 91$, $136.94,136.45,128.90,128.44,128.00,122 \cdot 83$, $122 \cdot 38,110 \cdot 29,55 \cdot 87,23 \cdot 14,21 \cdot 12$; IR (KBr plate, $\left.\mathrm{CDCl}_{3}, \mathrm{~cm}^{-1}\right) v=3254,2955,1665,1519$; MS (EI): $m / z=255$, 213. Anal. Calcd. for $\mathbf{C}_{16} \mathrm{H}_{17} \mathrm{NO}_{2}$ : C, 75.27; H, 6.71; N, 5.49. Found: C, 75.19; H, 6.88; $\mathrm{N}, 5 \cdot 63$.

$2.1 \mathrm{k} \quad \mathrm{N}-\left(4,4^{\prime}, 5\right.$-Trimethyl-biphenyl-2-yl)-acetamide (3ab): White solid; m.p.: $122-124^{\circ} \mathrm{C}$; ${ }^{1} \mathrm{H}$ NMR $\left(300 \mathrm{MHz}, \mathrm{CDCl}_{3}\right): \delta=7.95(s, 1 \mathrm{H}, \mathrm{NH}), 7.24(t$, $J=8.4 \mathrm{~Hz}, 4 \mathrm{H}), 7.07(s, 1 \mathrm{H}), 7.01(d, J=14.4 \mathrm{~Hz}$, $1 \mathrm{H}), 2.40(s, 3 \mathrm{H}), 2.29(s, 3 \mathrm{H}), 2.24(s, 3 \mathrm{H}), 2.00(s$, $3 \mathrm{H}) ;{ }^{13} \mathrm{C}$ NMR $\left(75 \mathrm{MHz}, \mathrm{CDCl}_{3}\right): \quad \delta=168.21$, $137 \cdot 32,136 \cdot 50,135 \cdot 26,132.78,132 \cdot 16,131.03$, $130 \cdot 11,129 \cdot 57,129 \cdot 04,123 \cdot 22,24 \cdot 39,21 \cdot 11,19 \cdot 68$, 19.16; IR (KBr plate, $\mathrm{CDCl}_{3}, \mathrm{~cm}^{-1}$ ) $v=3275,3022$, 1668, 1522; MS (EI): $m / z=253,211$. Anal. Calcd. for $\mathrm{C}_{17} \mathrm{H}_{19} \mathrm{NO}$ : $\mathrm{C}, 80 \cdot 60 ; \mathrm{H}, 7.56 ; \mathrm{N}, 5 \cdot 53$. Found: $\mathrm{C}$, $80 \cdot 72 ; \mathrm{H}, 7.69 ; \mathrm{N}, 5 \cdot 59$.

$2.11 \mathrm{~N}$-(4-Chloro-4'methyl-biphenyl-2-yl)-acetamide (3eb): White solid; ${ }^{1} \mathrm{H}$ NMR $(300 \mathrm{MHz}$, $\left.\mathrm{CDCl}_{3}\right): \delta=8.40(s, 1 \mathrm{H}, \mathrm{NH}), 7.29(d, J=8.40 \mathrm{~Hz}$, $2 \mathrm{H}), 7 \cdot 21(d, J=8 \cdot 10 \mathrm{~Hz}, 3 \mathrm{H}), 7 \cdot 12(s, 2 \mathrm{H}), 2.42(s$, $3 \mathrm{H}), 2.01(s, 3 \mathrm{H}) ;{ }^{13} \mathrm{C}$ NMR $\left(75 \mathrm{MHz}, \mathrm{CDCl}_{3}\right)$ : $\delta=168 \cdot 14,138 \cdot 23,135 \cdot 81,134 \cdot 04,133 \cdot 86,131 \cdot 86$, $130 \cdot 87,129.98,128.98,124 \cdot 16,121 \cdot 00,24 \cdot 6121 \cdot 18$; IR ( $\mathrm{KBr}$ plate, $\mathrm{CDCl}_{3}, \mathrm{~cm}^{-1}$ ) $v=3273,2920,1668$, 1517; MS (EI): $m / z=261,259,217$. Anal. Calcd. for $\mathrm{C}_{15} \mathrm{H}_{14} \mathrm{ClNO}$ : $\mathrm{C}, 69 \cdot 36 ; \mathrm{H}, 5 \cdot 43 ; \mathrm{N}, 5 \cdot 39$. Found: C, 69.51; H, 4.60; N, 5.23.

$2.1 \mathrm{~m} \quad N-(3,4,5-T r i m e t h y l-b i p h e n y l-2-y l)$-acetamide (3ac): White solid; m.p.: $92-93^{\circ} \mathrm{C}$; ${ }^{1} \mathrm{H}$ NMR $\left(300 \mathrm{MHz}, \mathrm{CDCl}_{3}\right): \delta=7.98(s, 1 \mathrm{H}, \mathrm{NH}), 7.34(t$, $J=7.2 \mathrm{~Hz}, 1 \mathrm{H}), 7.21-7.09(m, 4 \mathrm{H}), 7.02(s, 1 \mathrm{H})$, $2.41(s, 3 \mathrm{H}), 2.31(s, 3 \mathrm{H}), 2 \cdot 25(s, 3 \mathrm{H}), 2 \cdot 01(s, 3 \mathrm{H})$; ${ }^{13} \mathrm{C}$ NMR $\left(75 \mathrm{MHz}, \mathrm{CDCl}_{3}\right): \delta=168.15,138.64$, $138.22,136.59,132.73,132 \cdot 12,130.95,130.23$, $129 \cdot 95,128 \cdot 67,128 \cdot 28,126 \cdot 10,123 \cdot 18,24 \cdot 38$,
21.40, 19.69, 19.14; IR ( $\mathrm{KBr}$ plate, $\left.\mathrm{CDCl}_{3}, \mathrm{~cm}^{-1}\right)$ $v=3271,3022,1665,1522$; MS (EI): $m / z=253$, 211. Anal. Calcd. for $\mathrm{C}_{17} \mathrm{H}_{19} \mathrm{NO}$ : C, 80.60; H, 7.56; N, 5.53. Found: C, 68.49; H, 7.43; N, 5.62.

2.1n N-(3'-Methyl-biphenyl-2-yl)-acetamide (3fc): White solid; ${ }^{1} \mathrm{H}$ NMR $\left(300 \mathrm{MHz}, \mathrm{CDCl}_{3}\right): \delta=8.27$ $(d, J=7.20 \mathrm{~Hz}, 1 \mathrm{H}, \mathrm{NH}), 7.39-7.33(m, 2 \mathrm{H}), 7.26-$ $7 \cdot 18(m, 6 \mathrm{H}), 2.41(s, 3 \mathrm{H}), 2 \cdot 01(s, 3 \mathrm{H}) ;{ }^{13} \mathrm{C}$ NMR $\left(75 \mathrm{MHz}, \mathrm{CDCl}_{3}\right): \delta=168 \cdot 19,138 \cdot 89,138 \cdot 04,134 \cdot 68$, $132,18 \quad 131 \cdot 78, \quad 129 \cdot 97, \quad 128 \cdot 87, \quad 128 \cdot 66,128 \cdot 27$, $126 \cdot 10,124 \cdot 21,121 \cdot 43,24 \cdot 59,21 \cdot 43$; IR (KBr plate, $\left.\mathrm{CDCl}_{3}, \mathrm{~cm}^{-1}\right) v=3291,3033,1687,1520$; MS (EI): $m / z=225,183$. Anal. Calcd. for $\mathrm{C}_{15} \mathrm{H}_{15} \mathrm{NO}: \mathrm{C}, 79.97$; H, 6.71; N, 6.22. Found: C, 80.13; H, 6.58; N, 6.40.

2.1o N-Acetyl-2,6-di-(3-chlorophenyl)-aniline (4fc): White solid; m.p.: $110-112^{\circ} \mathrm{C} ;{ }^{1} \mathrm{H}$ NMR $(300 \mathrm{MHz}$, $\left.\mathrm{CDCl}_{3}\right): \delta=7 \cdot 42-7.28(m, 4 \mathrm{H}), 7 \cdot 21-7 \cdot 14(m, 7 \mathrm{H})$, $6.55(s, 1 \mathrm{H}, \mathrm{NH}), 2.41(s, 6 \mathrm{H}), 1.70(s, 3 \mathrm{H}) ;{ }^{13} \mathrm{C}$ NMR $\left(75 \mathrm{MHz}, \mathrm{CDCl}_{3}\right): \delta=69 \cdot 17,140 \cdot 81,139 \cdot 76$, $137.77,131.18,130 \cdot 19,129.80,129.48,127.98$, $127 \cdot 60,125 \cdot 72,22 \cdot 89,21 \cdot 46$; IR (KBr plate, $\mathrm{CDCl}_{3}$, $\left.\mathrm{cm}^{-1}\right) \quad v=3241,3027,1657,1526$; MS (EI): $\mathrm{m} / \mathrm{z}=$ 315,273 . Anal. Calcd. for $\mathrm{C}_{22} \mathrm{H}_{21} \mathrm{NO}: \mathrm{C}, 83.78 ; \mathrm{H}$, $6 \cdot 71 ; \mathrm{N}, 4.44$. Found: C, 83.62; H, 6.86; N, 4.53.

2.1p N-(4'-Methoxy-4,5-dimethyl-biphenyl-2-yl)acetamide (3ad): White solid; m.p.: $136-138^{\circ} \mathrm{C} ;{ }^{1} \mathrm{H}$ NMR (300 MHz, $\left.\mathrm{CDCl}_{3}\right): \delta=7.95(s, 1 \mathrm{H}, \mathrm{NH}), 7.27$ $(d, J=8.7 \mathrm{~Hz}, 2 \mathrm{H}), 7.05-6.97(m, 4 \mathrm{H}), 3.85(s, 3 \mathrm{H})$, $2.95(s, 3 \mathrm{H}), 2.24(s, 3 \mathrm{H}), 2.01(s, 3 \mathrm{H}) ;{ }^{13} \mathrm{C}$ NMR $\left(75 \mathrm{MHz}, \mathrm{CDCl}_{3}\right): \quad \delta=168 \cdot 16, \quad 159 \cdot 05, \quad 136 \cdot 38$, $132.78,132 \cdot 25,131.07,130.44,130 \cdot 31,129.87$, $123 \cdot 21,114 \cdot 28,55 \cdot 26,24 \cdot 40,19 \cdot 66,19 \cdot 14$; IR (KBr plate, $\left.\mathrm{CDCl}_{3}, \mathrm{~cm}^{-1}\right) v=3289,1674,1517$; $\mathrm{MS}(\mathrm{EI})$ : $m / z=269$, 227. Anal. Calcd. for $\mathrm{C}_{17} \mathrm{H}_{19} \mathrm{NO}_{2}$ : C, $75 \cdot 81 ; \mathrm{H}, 7 \cdot 11 ; \mathrm{N}, 5 \cdot 20$. Found: C, 75.94; H, 7.01; $\mathrm{N}, 5 \cdot 30$.

2.1q $N$-(4'-Ethoxy-4,5-dimethyl-biphenyl-2-yl)acetamide (3ae): White solid; m.p.: $102-103^{\circ} \mathrm{C}$; ${ }^{1} \mathrm{H}$ NMR $\left(300 \mathrm{MHz}, \mathrm{CDCl}_{3}\right): \delta=7.95(s, 1 \mathrm{H}, \mathrm{NH})$, $7.25(d, J=8.4 \mathrm{~Hz}, 2 \mathrm{H}), 7.07-6.95(m, 4 \mathrm{H}), 4.08$ $(d d, J=6 \cdot 9,6 \cdot 9 \mathrm{~Hz}, 2 \mathrm{H}), 2 \cdot 29(s, 3 \mathrm{H}), 2 \cdot 24(s, 3 \mathrm{H})$, $2.01(s, 3 \mathrm{H}) 1.45(t, J=6.9 \mathrm{~Hz}, 3 \mathrm{H}) ;{ }^{13} \mathrm{C}$ NMR $\left(75 \mathrm{MHz}, \quad \mathrm{CDCl}_{3}\right): \quad \delta=168 \cdot 16, \quad 158.42, \quad 136.31$, $132.74,132 \cdot 24,131.06,130 \cdot 28,129.92,123 \cdot 18$, $114.78,63.45,24.39,19 \cdot 66,19 \cdot 14,14.78$; IR (KBr plate, $\left.\mathrm{CDCl}_{3}, \mathrm{~cm}^{-1}\right) v=3286,1669,1518$; $\mathrm{MS}(\mathrm{EI})$ : $m / z=283$, 241. Anal. Calcd. for $\mathrm{C}_{18} \mathrm{H}_{21} \mathrm{NO}_{2}$ : C, 
Table 1. Ortho arylation of acetanilide under different conditions ${ }^{\mathrm{a}}$

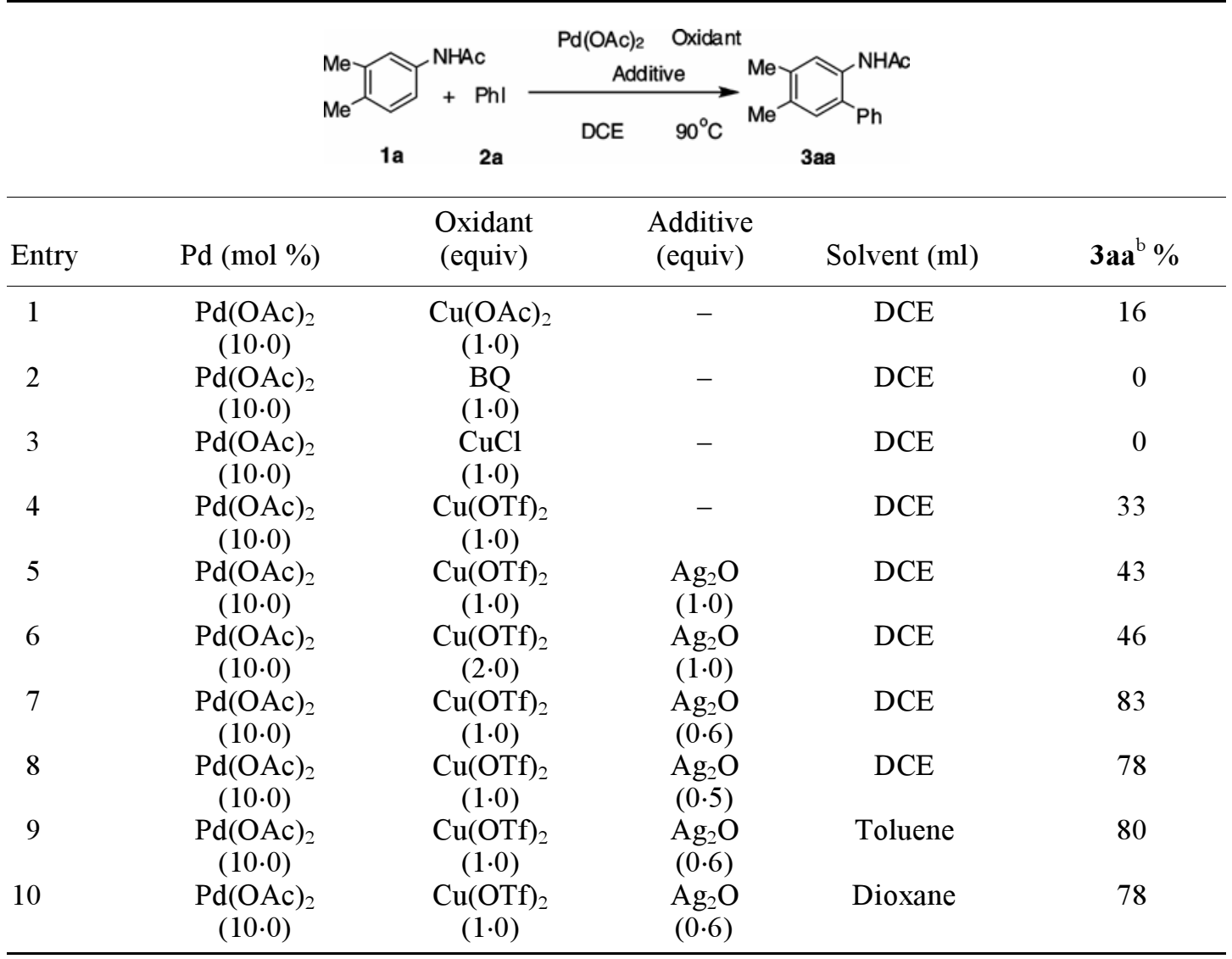

${ }^{a}$ All the reactions were carried out in the presence of $0.1 \mathrm{mmol}$ of $1 \mathrm{a}$ and $0.5 \mathrm{mmol} 2 \mathrm{a}$ in the solvent mentioned in each entry $(1.0 \mathrm{~mL})$ at $90^{\circ} \mathrm{C}$ for $14 \mathrm{~h}$. ${ }^{\text {bisolated yields }}$

76.29; H, 7.47; N, 4.94. Found: C, 76.44; H, 7.61; $\mathrm{N}, 4 \cdot 81$.

2.1r N-(4-Chloro-4'-ethoxy-biphenyl-2-yl)-acetamide (3ee): White solid; m.p.: $132-134{ }^{\circ} \mathrm{C} ;{ }^{1} \mathrm{H}$ NMR $\left(300 \mathrm{MHz}, \mathrm{CDCl}_{3}\right): \delta=8 \cdot 38(s, 1 \mathrm{H}, \mathrm{NH}), 7 \cdot 26-7 \cdot 12$ $(m, 5 \mathrm{H}), 7.02(m, 2 \mathrm{H}), 4.09(d d, J=7.20 \mathrm{~Hz}, 2 \mathrm{H})$, $2.02(s, 3 \mathrm{H}), 1.43(t, J=7.20 \mathrm{~Hz}, 3 \mathrm{H}) ;{ }^{13} \mathrm{C}$ NMR $\left(75 \mathrm{MHz}, \quad \mathrm{CDCl}_{3}\right): \quad \delta=168 \cdot 11, \quad 158.94, \quad 135.85$, $133.64,130 \cdot 91,130 \cdot 28,129 \cdot 79,128 \cdot 86,124 \cdot 08$, $120.91,115 \cdot 15,63 \cdot 57,24 \cdot 65,14 \cdot 79$; IR (KBr plate, $\left.\mathrm{CDCl}_{3}, \mathrm{~cm}^{-1}\right) v=3301,2920,1666,1517$; $\mathrm{MS}(\mathrm{EI})$ : $m / z=291,289,247$. Anal. Calcd. for $\mathrm{C}_{16} \mathrm{H}_{16} \mathrm{ClNO}_{2}$ : $\mathrm{C}, 66.32 ; \mathrm{H}, 5.57 ; \mathrm{N}, 4.83$. Found: $\mathrm{C}, 66.49 ; \mathrm{H}$, $5 \cdot 41 ; \mathrm{N}, 5 \cdot 01$.

2.1s N-(3'-Chloro-4,5-dimethyl-biphenyl-2-yl)-acetamide (3af): White solid; m.p.: $106-108^{\circ} \mathrm{C}$; ${ }^{1} \mathrm{H}$ NMR $\left(300 \mathrm{MHz}, \mathrm{CDCl}_{3}\right): \delta=7.83(s, 1 \mathrm{H}, \mathrm{NH})$, 7.37-7.34 (m, 3H), 7.26-7.22 ( $m, 1 \mathrm{H}), 7.00(s, 2 \mathrm{H})$, $2.29(s, 3 \mathrm{H}), 2.25(s, 3 \mathrm{H}), 2.01(s, 3 \mathrm{H}) ;{ }^{13} \mathrm{C} \mathrm{NMR}$ $\left(75 \mathrm{MHz}, \quad \mathrm{CDCl}_{3}\right): \quad \delta=168.41, \quad 140.27, \quad 137.32$,
$133.41,131.84,130.86,129.95,129.43,129.31$, $127.63,127 \cdot 27,124 \cdot 25,109 \cdot 71,24.23,19 \cdot 71,19 \cdot 17$; IR ( $\mathrm{KBr}$ plate, $\left.\mathrm{CDCl}_{3}, \mathrm{~cm}^{-1}\right) \quad v=3259,3022,1662$, 1524; MS (EI): $m / z=275,273,231$. Anal. Calcd. for $\mathrm{C}_{16} \mathrm{H}_{16} \mathrm{ClNO}$ : C, 70.20; H, 5.89; N, 5.12. Found: C, $70 \cdot 36 ; \mathrm{H}, 5 \cdot 78 ; \mathrm{N}, 5 \cdot 16$.

2.1t $\quad N$-(4'-Iodo-4,5-dimethyl-biphenyl-2-yl)-acetamide (3ag): White solid; m.p.: $176-178^{\circ} \mathrm{C} ;{ }^{1} \mathrm{H}$ NMR (300 MHz, $\left.\mathrm{CDCl}_{3}\right): \delta=7.85(s, 1 \mathrm{H}, \mathrm{NH}), 7 \cdot 77$ $(d, J=8.40 \mathrm{~Hz}, 2 \mathrm{H}), 7.09(d, J=8.40 \mathrm{~Hz}, 2 \mathrm{H}), 6.96$ $(d, \mathbf{J}=10 \cdot 80,2 \mathrm{H}), 2.29(s, 3 \mathrm{H}), 2.25(s, 3 \mathrm{H}), 2.02(s$, $3 \mathrm{H}) ;{ }^{13} \mathrm{C} \quad \mathrm{NMR}\left(75 \mathrm{MHz}, \mathrm{CDCl}_{3}\right): \quad \delta=168.39$, $137.91,137.21,133.44,131.79,131.06,130.81$, $129 \cdot 70,124 \cdot 24,93 \cdot 32,24 \cdot 28,19 \cdot 71,19 \cdot 18$; IR $(\mathrm{KBr}$ plate, $\left.\mathrm{CDCl}_{3}, \mathrm{~cm}^{-1}\right) v=3254,3020,1653,1525$; MS (EI): $m / z=365$, 323. Anal. Calcd. for $\mathrm{C}_{16} \mathrm{H}_{16} \mathrm{INO}$ : C, $52.62 ; \mathrm{H}, 4.42 ; \mathrm{N}, 3.84$. Found: C, 52.74; H, 4.33; $\mathrm{N}, 4 \cdot 01$.

2.1u N-(4'-Iodo-3-methyl-biphenyl-2-yl)-acetamide (3cg): White solid; m.p.: $148-150^{\circ} \mathrm{C} ;{ }^{1} \mathrm{H}$ NMR 
Table 2. Arylation of acetanilides. ${ }^{\mathrm{a}}$

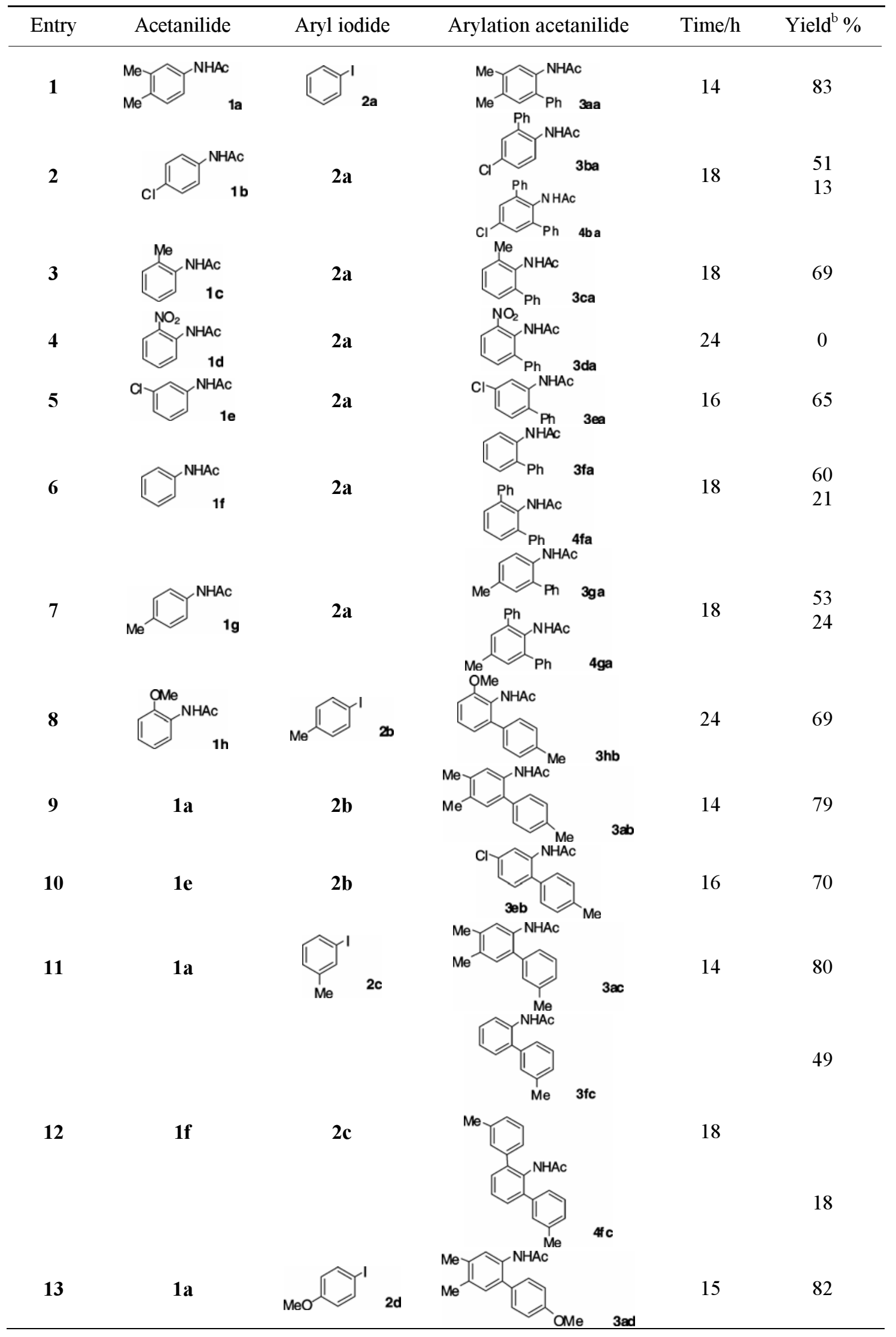


Table 2. (Contd ...

\begin{tabular}{|c|c|c|c|c|c|}
\hline Entry & Acetanilide & Aryl iodide & Arylation acetanilide & Time/h & Yield $^{\mathrm{b}} \%$ \\
\hline 14 & $1 \mathbf{a}$ & & & 15 & 80 \\
\hline 15 & 1e & $2 e$ & & 16 & 73 \\
\hline 16 & 1a & & & 15 & 67 \\
\hline 17 & 1a & & & 24 & 49 \\
\hline 18 & 1c & $2 g$ & & 24 & 40 \\
\hline
\end{tabular}

${ }^{a}$ All the reactions were carried out with acetanilide $1(0.2 \mathrm{mmol})$ and aryl iodide $2(1.0 \mathrm{mmol})$ in the presence of $\mathrm{Pd}(\mathrm{OAc})_{2}(10 \mathrm{~mol} \%), \mathrm{Cu}(\mathrm{OTf})_{2}\left(1.0\right.$ equiv), and $\mathrm{Ag}_{2} \mathrm{O}$ (0.6 equiv) in $\mathrm{DCE}(2 \mathrm{ml})$ at $90^{\circ} \mathrm{C}$. ${ }^{\text {bisolated yields }}$

$\left(300 \mathrm{MHz}, \mathrm{CDCl}_{3}\right): \delta=7.72(d, J=8.40 \mathrm{~Hz}, 2 \mathrm{H})$, 7.31-7.25 (m, 2H), 7.14-7.11 (m, 1H), 7.06 (d, $J=8.40 \mathrm{~Hz}, 2 \mathrm{H}), 6.66(s, 1 \mathrm{H}, \mathrm{NH}), 2.29(s, 3 \mathrm{H})$, $2.01(s, 3 \mathrm{H}) ;{ }^{13} \mathrm{C} \mathrm{NMR}\left(75 \mathrm{MHz}, \mathrm{CDCl}_{3}\right): \delta=69 \cdot 34$, $139.16,138.67,137.39,137.03,132.34,130.72$, $130.46,128.46,127 \cdot 65,94 \cdot 25,23.04,18 \cdot 54 ;$ IR $\left(\mathrm{KBr}\right.$ plate, $\left.\mathrm{CDCl}_{3}, \mathrm{~cm}^{-1}\right) \quad v=3246,3022,1646$, 1524; MS (EI): $m / z=351,309$. Anal. Calcd. For $\mathrm{C}_{15} \mathrm{H}_{14} \mathrm{INO}$ : C, 51.30; H, 4.02; N, 3.99. Found: C, $51 \cdot 46 ; \mathrm{H}, 4 \cdot 17$; N, 3.84.

\section{Results and discussion}

In our initial screening experiments, acetanilide (1a) and iodobenzene (2a) were used as the prototype substrates for optimizing the reaction conditions. When $1 \mathbf{a}$ and $\mathbf{2 a}$ were catalysed with $\mathrm{Pd}(\mathrm{OAc})_{2}$ in the presence of $\mathrm{Cu}(\mathrm{OAc})_{2}$, 3aa was isolated in low yield (table 1 , entry 1 ). It was found that $\mathrm{Cu}(\mathrm{OTf})_{2}$ was more effective than $\mathrm{Cu}(\mathrm{OAc})_{2}$ as oxidant for this transformation (table 1 , entry 4 ). We studied the effect of additive and solvent on this reaction. When 1a was treated with $\mathbf{2 a}$ under the conditions utilized by Shi group for the arylation of $\mathrm{N}$-alkyl acetanilides, compound 3 aa was only isolated in $43 \%$ yield (table 1, entry 5). Further studies indicated that the amount of $\mathrm{Ag}_{2} \mathrm{O}$ was very important for this reaction. A $83 \%$ yield of 3 aa was obtained when the amount of $\mathrm{Ag}_{2} \mathrm{O}$ was decreased to $60 \mathrm{~mol} \%$ (table 1, entry 7). Among the solvents tried, 1,2-dichloroethane (DCE) proved to be the most suitable (table 1, entry 7,9 and 10).

The optimized reaction conditions were applied for the arylation of a number of differently substituted acetanilides with a variety of aryl iodides (table 2). Of the aromatic aryl iodides investigated, 1,4-diiodobenzene (2. g) gave the lowest yield of arylated product (table 2, entry 17, 18). On the other hand, acetanilides substituted in 2-or 3-positions only afforded monoarylated products. Both monoarylation and diarylation products were attained, when acetanilide (1f) and 4-substituted acetanilides were used as substrates (table 2, entry 2, 6, 7, 12). We found that 2-nitroacetanilide did not undergo arylation under the standard reaction conditions (table 2, entry 4)

\section{Conclusions}

In conclusion, a direct and efficient method for the ortho arylation of acetanilides has been developed. A number of acetanilides were coupled with aryl iodides to afford the corresponding products in moderate to high yields. Further investigations on the scope and synthetic applications of this reaction are in progress. 


\section{Acknowledgement}

The authors thank State Key Laboratory of Applied Organic Chemistry for financial support.

\section{References}

1. (a) Hassan J, Sevignon M, Gozzi C, Schulz E and Lemaire M 2002 Chem. Rev. 102 1359; (b) Storr T E, Firth A G, Wilson K, Darley K, Baumann C G, Fairlamb I J S 2008 Tetrahedron 646125

2. (a) Stanforth S P 1998 Tetrahedron 54 263; (b) Bressy C, Alberico D and Lautens M $2005 \mathrm{~J}$. Am. Chem. Soc. 12713148

3. (a) Alberico D, Scott M E and Lautens M 2007 Chem. Rev. 107 174; (b) Campo M A, Huang Q, Yao T, Tian Q and Larock R C $2003 \mathrm{~J}$. Am. Chem. Soc. 125 11506; (c) Chen X, Li J-J, Hao X-S, Goodhue C E and Yu J-Q 2006 J. Am. Chem. Soc. 128 78; (d) Lafrance M, Shore D and Fagnou K 2006 Org. Lett. 8 5097; (e) Li W, Nelson D P, Jensen M S, Hoerrner R S, Javadi G J, Cai D and Larsen R D 2003 Org. Lett. 54835

4. (a) Kametani Y, Satoh T, Miura M and Nomura M 2000 Tetrahedron Lett. 41 2655; (b) Tsang W C P, Zheng N and Buchwald S L $2005 \mathrm{~J}$. Am. Chem. Soc.
127 14560; (c) Tremont S J and Rahman H U $1984 J$. Am. Chem. Soc. 1065759

5. (a) Oi S, Aizawa E, Ogino Y and Inoue Y $2005 \mathrm{~J}$. Org. Chem. 70 3113; (b) Giri R, Chen X and Yu J-Q 2005 Angew. Chem. Int. Ed. 442112

6. (a) Kalyani D, Deprez N R, Desai L V and Sanford M S 2005 J. Am. Chem. Soc. 127 7330; (b) Oi S, Fukita $\mathrm{S}$, Hirata N, Watanuki N, Miyano S and Inoue Y 2001 Org. Let. 3 2579; (c) Ackermann L 2005 Org. Lett. 143123

7. Larivee A, Mousseau J J and Charette A B $2008 J$. Am. Chem. Soc. 13052

8. (a) Dupont J, Consorti C S and Spencer J 2005 Chem. Rev. 105 2527; (b) Wang Z, Zhang Z and Lu X 2000 Organometallics 19775 ; (c) Horino $\mathrm{H}$ and Inoue N 1981 J. Org. Chem. 46 4416; (d) Zaitsev V G and Daugulis O $2005 \mathrm{~J}$. Am. Chem. Soc. 127 4156; (e) Boele M D K, van Strijdonck G P F, de Vries A H M, Kamer P C J, de Vries J G and van Leeuwen P W N M 2002 J. Am. Chem. Soc. 1241586 (f) Shi Z, Li B, Wan X, Cheng J, Fang Z, Cao B, Qin C and Wang Y 2007 Angew. Chem. Int. Ed. 465554 (g) Yang S, Li B, Wan X and Shi Z 2007 J. Am. Chem. Soc. 129 6066; (h) Wan X, Ma Z, Li B, Zhang K, Cao S, Zhang S and Shi Z 2006 J. Am. Chem. Soc. 128 7416; (i) Shabashov D and Daugulis O $2007 \mathrm{~J}$. Org. Chem. 72 7720; (j) Daugulis O and Zaitsev V G 2005 Angew. Chem., Int. Ed. 444046 